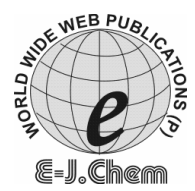

http://www.e-journals.net
ISSN: 0973-4945; CODEN ECJHAO

E-Journal of Chemistry

Vol. 5, No.4, pp.688-695, October 2008

\title{
An Efficient and Recycling Catalyst for the One-Pot Three-Component Synthesis of Substituted 3,4-Dihydropyrimidin-2(1H)-ones
}

\author{
TAOUES BOUMOUD ${ }^{\S}$, BOUDJEMAA BOUMOUD ${ }^{\S}$, \\ SALAH RHOUATI ${ }^{\S}$, ALI BELFAITAH ${ }^{\S}$, \\ ABDELMADJID DEBACHE ${ }^{{ }^{*}}$ and PAUL MOSSET ${ }^{\#}$ \\ ${ }^{\S}$ Laboratoire des Produits Naturels d'origine Végétale et de Synthèse \\ Organique. Département de Chimie. Faculté des Sciences. \\ Université Mentouri de Constantine 25000 Constantine, Algérie.

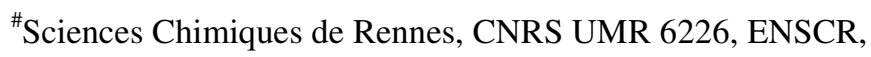 \\ Avenue du Général Leclerc, 35700 Rennes, France. \\ ta_boumoud@yahoo.fr; Tel.: (213) 72386009
}

Received 3 December 2007; Accepted 1 February 2008

\begin{abstract}
The Biginelli one-pot three-component cyclocondensation was applied in this work to prepare 3,4-dihydropyrimidinone and its analogues using the first derivative of lead, $\mathrm{Pb}\left(\mathrm{NO}_{3}\right)_{2}$, as a recycling catalyst, from a diversity of aromatic aldehydes, $\beta$-ketoesters and urea. The reaction was carried out in refluxing acetonitrile and afforded the target molecules in good to excellent yields. The method offers several advantages including high yields of the products, short reaction times and easy experimental workup procedure.
\end{abstract}

Keywords: Biginelli reaction, multi-component reactions (MCRs), Dihydropyrimidinones (DHPMs), Lead(II) nitrate, Scaffold, Leader file.

\section{Introduction}

Heterocyclic moiety is an important structure in many bioactive natural products and therapeutic compounds. In view of the increasing interest for the preparation of large heterocyclic compounds libraries and beside the usual multi-step syntheses, multicomponent reactions (MCRs) are becoming increasingly prevalent due to their improved efficiency, simple procedure, one-pot character, quantitative yields of the target molecules and the high and ever increasing number of accessible backbones. 
3,4-Dihydropyrimidin-2-(1H)-one (DHPM) first synthesized by the original multicomponent one-pot Biginelli reaction ${ }^{1}$ in 1893, and its derivatives show a diverse range of therapeutical properties and pharmacological activities ${ }^{2}$ such as antimitotic ${ }^{3}$, analgesic ${ }^{4}$, antiviral $^{5}$, anticancer ${ }^{6}$, anti-inflammatory ${ }^{2,4,7}$ and antihypertensive agents ${ }^{8}$. Noteworthy, they have served as integral backbones of several calcium channel modulators ${ }^{7}$. DHPMs were also screened as neuropeptide antagonists ${ }^{8}$, agents in treating anxiety ${ }^{9}$, optic nerve dysfunction $^{10}$ and recently as antioxydant agents ${ }^{11}$. Further, the DHPMs scaffold is contained in a number of natural products including batzelladine alkaloids $\mathrm{A}$ and $\mathrm{B}$ which are found to inhibit the binding of HIVgp-120-CD4 cells ${ }^{12,13}$.

Due to the importance of MCRs in combinatorial chemistry and the interesting pharmacological properties associated with DHPMs structures, the Biginelli reaction has received increasing attention and its scope has now extended considerably by variation of all three building blocks, thus, several modified and improved procedures have been reported ${ }^{14}$.

Among the diversity of methodologies reported in the literature, special attention has been dedicated to:

-Lewis acids, namely, $\mathrm{Yb}(\mathrm{OTf})_{3}{ }^{15}, \mathrm{InCl}_{3}{ }^{16}, \mathrm{VCl}_{3}{ }^{17}, \mathrm{CuCl}_{2 .} 2 \mathrm{H}_{2} \mathrm{O}^{18}, \mathrm{LiBr}^{19}, \mathrm{LiClO}_{4}{ }^{20}$, $\mathrm{RuCl}_{3}{ }^{21}, \mathrm{SnCl}_{2} \cdot 2 \mathrm{H}_{2} \mathrm{O}^{22}, \mathrm{BF}_{3} \cdot \mathrm{OEt}_{2}{ }^{23}, \mathrm{ZrCl}_{4}{ }^{24}, \quad \mathrm{Y}\left(\mathrm{NO}_{3}\right)_{3} \cdot 6 \mathrm{H}_{2} \mathrm{O}^{25}, \mathrm{Cu}(\mathrm{OTf})_{2}{ }^{26}, \mathrm{CuI}^{27}$, $\mathrm{InBr}_{3}{ }^{28}, \mathrm{~B}(\mathrm{OH})_{3}{ }^{29}, \mathrm{In}(\mathrm{OTf})_{3}{ }^{30}, \mathrm{PhB}(\mathrm{OH})_{2}{ }^{31}, \mathrm{Fe}(\mathrm{OAc})_{3}, \mathrm{Fe}(\mathrm{OTf})_{3}{ }^{32}$ and $\mathrm{HBF}_{4}{ }^{33}$.

-Brônsted acids such as $p$-toluenesulfonic $\operatorname{acid}^{34}$, silica sulphuric $\operatorname{acid}^{35}$, potassium hydrogen sulphate ${ }^{36}$, formic acid ${ }^{37}$ and chloroacetic acid ${ }^{38}$.

-Heteropoly acids such as 12-molybdophosphoric acid $\mathrm{H}_{3} \mathrm{PMo}_{12} \mathrm{O}_{40}{ }^{39}$ and 11-molybdo1-vanadophosphoric acid $\mathrm{H}_{4} \mathrm{PMo}_{11} \mathrm{VO}_{40}{ }^{40}$.

Even bakers' yeast has been used as an efficient catalyst in Biginelli reaction ${ }^{41}$.

Moreover, asymmetric syntheses of DHPMs using $\mathrm{CeCl}_{3} / \mathrm{InCl}_{3}$ or $\mathrm{Yb}(\mathrm{OTf})_{3}$ as catalysts in the presence of chiral ligands have been reported ${ }^{42}$. These reactions can be carried out in ionic liquids ${ }^{43}$, under solid or fluorous phase ${ }^{44,45}$, under microwave with polyphosphate ester ${ }^{46}$ or ultrasound irradiations in the presence of $\mathrm{NH}_{2} \mathrm{SO}_{3} \mathrm{H}^{47}$ or $\mathrm{Mg}\left(\mathrm{ClO}_{4}\right)_{2}{ }^{48}$.

However, in spite of their potential utility, many of these methods generally require strong acidic conditions, stoichiometric amount of the catalysts, expensive reagents, prolonged reaction times and high temperatures. Thus, to avoid these limitations, we describe in this report an effective and rapid method for the preparation of DHPMs using a new catalytic agent $\mathrm{Pb}\left(\mathrm{NO}_{3}\right)_{2}$ in refluxed acetonitrile, (Scheme 1)

\section{Experimental}

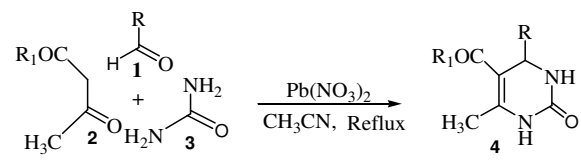

Scheme 1

\section{Maretials and methods}

Melting points were measured using a fine control Electro thermal capillary apparatus and are uncorrected. ${ }^{1} \mathrm{H}$ and ${ }^{13} \mathrm{C}$ NMR spectra were recorded on a BRUKER AVANCE DPX spectrometer at 250 and $62.9 \mathrm{MHz}$, respectively. NMR spectra were obtained on solutions in DMSO- $d_{6}$. Chemical shifts are reported in parts of million $(\delta \mathrm{ppm})$ relative to TMS $(\delta 0.0)$ as internal standard and coupling constant $(J)$ is reported in hertz (Hz). IR spectra were obtained as potassium bromide (KBr) pellets with a Shimadzu FT IR-8201 PC spectrometer. 


\section{General procedure}

A mixture of aldehyde $(1.0 \mathrm{mmol}), \beta$-ketoester $(1.0 \mathrm{mmol})$, urea $(1.5 \mathrm{mmol})$ and a catalytic amount of $\mathrm{Pb}\left(\mathrm{NO}_{3}\right)_{2}(5 \mathrm{~mol} \%)$ was refluxed in acetonitrile $(3 \mathrm{~mL})$ under magnetic stirring for the appropriate time as indicated in Table 1. Upon completion of the reaction, as indicated by TLC, the reaction mixture was cooled to room temperature, poured onto crushed ice and additionally stirred for several minutes.

The resulting solid was filtered under suction, washed with cold ethanol $(4 \mathrm{~mL})$ and recrystallized from hot ethanol to afford the pure product. In most cases, the crude product was dried under vacuum pump and shown essentially the same purity as the recrystallized sample. Finally, the aqueous phase was evaporated, and the catalyst $\mathrm{Pb}\left(\mathrm{NO}_{3}\right)_{2}$ was recovered.

All compounds obtained according to this protocol were characterized and identified by their melting points and NMR spectra in comparison to those reported in the literature. The results are summarised in Table 1.

\section{Physical and spectral data for all the compounds}

5-Ethoxycarbonyl-6-methyl-4-phenyl-3,4-dihydropyrimidin-2(1H)-one: $4 a$

M.p. $206-207^{\circ} \mathrm{C},{ }^{1} \mathrm{H}$ NMR (DMSO-d $\left.{ }_{6}\right): \delta(\mathrm{ppm})=9.20(\mathrm{~s}, 1 \mathrm{H}, \mathrm{NH}), 7.78(\mathrm{~s}, 1 \mathrm{H}, \mathrm{NH}), 7.28$ $\left(\mathrm{s}, 5 \mathrm{H}, \mathrm{C}_{6} \mathrm{H}_{5}\right), 5.14(\mathrm{~s}, 1 \mathrm{H}, \mathrm{CH}), 3.97$ (q, $\left.J=7.06 \mathrm{~Hz}, 2 \mathrm{H}, \mathrm{OCH}_{2} \mathrm{CH}_{3}\right), 2.25\left(\mathrm{~s}, 3 \mathrm{H}, \mathrm{CH}_{3}\right)$, $1.09\left(\mathrm{t}, J=7.06 \mathrm{~Hz}, 3 \mathrm{H}, \mathrm{OCH}_{2} \mathrm{CH}_{3}\right) ;{ }^{13} \mathrm{C} \mathrm{NMR}\left(\mathrm{DMSO}-\mathrm{d}_{6}\right): \delta(\mathrm{ppm})=165.7,152.6,148.8$, 145.3, 128.8, 127.7, 126.7, 106,4, 59.6, 54.4, 18.2, 14.5; IR (KBr) $\left(v_{\max } \mathrm{cm}^{-1}\right) 3242,3117$, 2980, 1721, 1637, 1522, 1462, 1288, 1092, 770.

\section{5-Ethoxycarbonyl-6-methyl-4-(2-methylphenyl)-3,4-dihydropyrimidin-2(1H)-one: $4 b$}

M.p. $202-204^{\circ} \mathrm{C},{ }^{1} \mathrm{H}$ NMR $\left(\mathrm{DMSO}_{\mathrm{d}}\right): \delta(\mathrm{ppm})=9.18(\mathrm{~s}, 1 \mathrm{H}, \mathrm{NH}), 7.82(\mathrm{~s}, 1 \mathrm{H}, \mathrm{NH}), 7.15-$ $7.11\left(\mathrm{~m}, 4 \mathrm{H}, \mathrm{C}_{6} \mathrm{H}_{4}\right), 5.39(\mathrm{~s}, 1 \mathrm{H}, \mathrm{CH}), 3.97\left(\mathrm{q}, J=7.06 \mathrm{~Hz}, 2 \mathrm{H}, \mathrm{OCH}_{2} \mathrm{CH}_{3}\right), 2.40(\mathrm{~s}, 3 \mathrm{H}$, $\left.\mathrm{C}_{6} \mathrm{H}_{4}-\mathrm{CH}_{3}\right), 2.28\left(\mathrm{~s}, 3 \mathrm{H}, \mathrm{CH}_{3}\right), 0.98\left(\mathrm{t}, J=7.06 \mathrm{~Hz}, 3 \mathrm{H}, \mathrm{OCH}_{2} \mathrm{CH}_{3}\right) ;{ }^{13} \mathrm{C}$ NMR $\left(\mathrm{DMSO}-\mathrm{d}_{6}\right) \delta$ $(\mathrm{ppm})=165.3,151.6,148.4,143.3,134.7,130.1,127.2,126.6,93.3,59.1,50.6,18.7,17.7$, 13.9; IR (KBr) $\left(v_{\max } \mathrm{cm}^{-1}\right) 3248,3117,2975,1722,1630,1532,1462,1283,1092,768$.

\section{5-Ethoxycarbonyl-4-(2-methoxyphenyl)-6-methyl-3,4-dihydropyrimidin-2(1H)-one: 4c}

M.p. $258-259^{\circ} \mathrm{C},{ }^{1} \mathrm{H}$ NMR $\left(\mathrm{DMSO}_{\mathrm{d}}\right): \delta(\mathrm{ppm})=9.12(\mathrm{~s}, 1 \mathrm{H}, \mathrm{NH}), 7.56(\mathrm{~s}, 1 \mathrm{H}, \mathrm{NH}), 7.33-$ $7.24\left(\mathrm{~m}, 1 \mathrm{H}, \mathrm{CH}_{\text {arom }}\right), 7.07-7.01\left(\mathrm{~m}, 2 \mathrm{H}, \mathrm{CH}_{\text {arom }}\right), 6.93-6.57\left(\mathrm{~m}, 1 \mathrm{H}, \mathrm{CH}_{\text {arom }}\right), 5.58(\mathrm{~s}, 1 \mathrm{H}, \mathrm{CH})$, $4.04\left(\mathrm{q}, J=7.04 \mathrm{~Hz}, 2 \mathrm{H}, \mathrm{OCH}_{2} \mathrm{CH}_{3}\right), 3.87\left(\mathrm{~s}, 3 \mathrm{H}, \mathrm{OCH}_{3}\right), 2.07\left(\mathrm{~s}, 3 \mathrm{H}, \mathrm{CH}_{3}\right), 1.06$ (t, $J=$ $\left.7.04 \mathrm{~Hz}, \quad 3 \mathrm{H}, \mathrm{OCH}_{2} \mathrm{CH}_{3}\right) ;{ }^{13} \mathrm{C} \mathrm{NMR}\left(\mathrm{DMSO}_{6}\right): \delta(\mathrm{ppm})=165.6,156.6,152.6,148.5$, $131.8,129.3,127.1,120.3,111.0,108.2,65.0,55.2,49.1,14.8,14.3 ; \mathrm{IR}(\mathrm{KBr})\left(v_{\max } \mathrm{cm}^{-1}\right)$ $3224,3109,2928,2848,1721,1677,1522,1432,1274,759$.

\section{5-Ethoxycarbonyl-6-methyl-4-(3-hydroxy-4-methoxyphenyl)-3,4-dihydropyrimidin-} 2(1H)-one: $4 d$

M.p. $186-188^{\circ} \mathrm{C} .{ }^{1} \mathrm{H}$ NMR $\left(\mathrm{DMSO}_{-} \mathrm{d}_{6}\right): \delta(\mathrm{ppm})=9.12,(\mathrm{~s}, 1 \mathrm{H}, \mathrm{OH}), 8.92(\mathrm{~s}, 1 \mathrm{H}, \mathrm{NH}), 7.61$ $(\mathrm{s}, 1 \mathrm{H}, \mathrm{NH}), 6.77-6.65\left(\mathrm{~m}, 3 \mathrm{H}, \mathrm{C}_{6} H_{3}\right), 5.04(\mathrm{~s}, 1 \mathrm{H}, \mathrm{CH}), 4.05(\mathrm{q}, J=7.04 \mathrm{~Hz}, 2 \mathrm{H}$, $\left.\mathrm{OCH}_{2} \mathrm{CH}_{3}\right) 3.72\left(\mathrm{~s}, 3 \mathrm{H}, \mathrm{OCH}_{3}\right), 2.24\left(\mathrm{~s}, 3 \mathrm{H}, \mathrm{CH}_{3}\right), 1.12\left(\mathrm{t}, J=7.04 \mathrm{~Hz}, 3 \mathrm{H}, \mathrm{OCH}_{2} \mathrm{CH}_{3}\right) ;{ }^{13} \mathrm{C}$ NMR $\left(\right.$ DMSO-d $\left.{ }_{6}\right): \delta(\mathrm{ppm})=165.8,152.7,148.2,147.3,146.7,138.0,117.3,114.1,112.4$, 100.1, 59.6, 56.1, 53.8, 18.2, 14.5; IR (KBr) $\left(v_{\max } \mathrm{cm}^{-1}\right) 3242,3117,2980,2906,1728,1639$, 1532, 1460, 1278, 1092, 767. 
5-Ethoxycarbonyl-4-(3-methylphenyl)6-methyl-3,4-dihydropyrimidin-2(1H)-one: 4e M.p. $228-230{ }^{\circ} \mathrm{C},{ }^{1} \mathrm{H}$ NMR $\left(\mathrm{DMSO}_{6}\right): \delta(\mathrm{ppm})=9.18(\mathrm{~s}, 1 \mathrm{H}, \mathrm{N} H), 7.82(\mathrm{~s}, 1 \mathrm{H}, \mathrm{NH}), 7.12-7.00$ $\left(\mathrm{m}, 1 \mathrm{H}, \mathrm{CH}_{\text {arom }}\right), 5.25(\mathrm{~s}, 1 \mathrm{H}, \mathrm{CH}), 3.97\left(\mathrm{q}, J=7.06 \mathrm{~Hz}, 2 \mathrm{H}, \mathrm{OCH}_{2} \mathrm{CH}_{3}\right), 2.30\left(\mathrm{~s}, 3 \mathrm{H}, \mathrm{C}_{6} \mathrm{H}_{4}\right.$ $\left.\mathrm{CH}_{3}\right), 2.20\left(\mathrm{~s}, 3 \mathrm{H}, \mathrm{CH}_{3}\right), 1.09\left(\mathrm{t}, J=7.06 \mathrm{~Hz}, 3 \mathrm{H}, \mathrm{OCH}_{2} \mathrm{CH}_{3}\right) ;{ }^{13} \mathrm{C} \mathrm{NMR}\left(\mathrm{DMSO}-\mathrm{d}_{6}\right): \delta(\mathrm{ppm})=$ 165.6, 153.9, 148.4, 144.6, 137.9, 128.9, 128.4, 127.4, 123.9, 114.8, 62.0, 54.6, 24.8, 17.1, 14.9; $\operatorname{IR}(\mathrm{KBr})\left(v_{\max } \mathrm{cm}^{-1}\right) 3252,3117,2980,2880,1725,1639,1520,1472,1285,1072,775$.

5-Ethoxycarbonyl-4-(4-methoxyphenyl)-6-methyl-3,4-dihydropyrimidin-2(1H)-one: $4 f$ M.p. $200-202^{\circ} \mathrm{C},{ }^{1} \mathrm{H}$ NMR (DMSO-d $\left.\mathrm{d}_{6}\right): \delta(\mathrm{ppm})=9.17(\mathrm{~s}, 1 \mathrm{H}, \mathrm{NH}), 7.68(\mathrm{~s}, 1 \mathrm{H}, \mathrm{NH}), 7.14$ $\left(\mathrm{d}, J=8.7 \mathrm{~Hz}, 2 \mathrm{H}, \mathrm{CH}_{\text {arom }}\right), 6.83\left(\mathrm{~d}, J=8.7 \mathrm{~Hz}, 2 \mathrm{H}, \mathrm{CH}_{\text {arom }}\right), 5.07(\mathrm{~s}, 1 \mathrm{H}, \mathrm{CH}), 4.01(\mathrm{q}, J=$ $\left.7.1 \mathrm{~Hz}, 2 \mathrm{H}, \mathrm{OCH}_{2} \mathrm{CH}_{3}\right), 3.70\left(\mathrm{~s}, 3 \mathrm{H}, \mathrm{OCH}_{3}\right), 2.23\left(\mathrm{~s}, 3 \mathrm{H}, \mathrm{CH}_{3}\right), 1.20(\mathrm{t}, J=7.1 \mathrm{~Hz}, 3 \mathrm{H}$, $\left.\mathrm{OCH}_{2} \mathrm{CH}_{3}\right) ;{ }^{13} \mathrm{C}$ NMR $\left(\mathrm{DMSO}-\mathrm{d}_{6}\right): \delta(\mathrm{ppm})=165.2,158.2,152.0,147.4,136.9,127.1$, 113.2, 99.5, 58.6, 54.6, 53.3, 17.5, 13.8; IR (KBr) $\left(v_{\max } \mathrm{cm}^{-1}\right) 3242,3109,2980,2848,1721$, $1677,1532,1472,1268,1112,759$.

4-(3-Chlorophenyl)-5-ethoxycarbonyl-6-methyl-3,4-dihydropyrimidin-2(1H)-one: $4 g$ M.p. $190-193^{\circ} \mathrm{C},{ }^{1} \mathrm{H}$ NMR $\left(\mathrm{DMSO}_{-} \mathrm{d}_{6}\right): \delta(\mathrm{ppm})=9.24(\mathrm{~s}, 1 \mathrm{H}, \mathrm{NH}), 7.77(\mathrm{~s}, 1 \mathrm{H}, \mathrm{NH}), 7.36-$ $7.17\left(\mathrm{~m}, 4 \mathrm{H}, \mathrm{C}_{6} H_{4}\right), 5.14(\mathrm{~s}, 1 \mathrm{H}, \mathrm{CH}), 3.98\left(\mathrm{q}, J=7.0 \mathrm{~Hz}, 2 \mathrm{H}, \mathrm{OCH}_{2} \mathrm{CH}_{3}\right), 2.24(\mathrm{~s}, 3 \mathrm{H}$, $\left.\mathrm{CH}_{3}\right), 1.08\left(\mathrm{t}, J=7.0 \mathrm{~Hz}, 3 \mathrm{H}, \mathrm{OCH}_{2} \mathrm{CH}_{3}\right) ;{ }^{13} \mathrm{C} \mathrm{NMR}\left(\mathrm{DMSO}_{6}\right): \delta(\mathrm{ppm})=165.2,151.9$, $148.9,147.2,132.9,130.4,127.2,126.2,124.9,98.7,59.3,53.6,17.8,14.0 ; \mathrm{IR}(\mathrm{KBr})\left(v_{\max }\right.$ $\mathrm{cm}^{-1}$ ) 3275,3167, 2990, 1781, 1687, 1572, 1492, 1300, 1192, 778.

5-Ethoxycarbonyl-6-methyl-4-(4-methylphenyl)-3,4-dihydropyrimidin-2(1H)-one: $4 \mathrm{~h}$

M.p. $213-215^{\circ} \mathrm{C},{ }^{1} \mathrm{H}$ NMR (DMSO-d6): $\delta(\mathrm{ppm})=9.15(\mathrm{~s}, 1 \mathrm{H}, \mathrm{NH}), 7.80(\mathrm{~s}, 1 \mathrm{H}, \mathrm{NH}), 7.10$ $\left(\mathrm{s}, 4 \mathrm{H}, \mathrm{C}_{6} \mathrm{H}_{4}\right), 5.09(\mathrm{~s}, 1 \mathrm{H}, \mathrm{CH}), 3.96\left(\mathrm{q}, J=7.1 \mathrm{~Hz}, 2 \mathrm{H}, \mathrm{OCH}_{2} \mathrm{CH}_{3}\right), 2.33\left(\mathrm{~s}, 3 \mathrm{H}, \mathrm{C}_{6} \mathrm{H}_{4}-\mathrm{CH}_{3}\right)$, $2.23\left(\mathrm{~s}, 3 \mathrm{H}, \mathrm{CH}_{3}\right), 1.08\left(\mathrm{t}, J=7.1 \mathrm{~Hz}, 3 \mathrm{H}, \mathrm{OCH}_{2} \mathrm{CH}_{3}\right) ;{ }^{13} \mathrm{C} \mathrm{NMR}\left(\mathrm{DMSO}_{6} \mathrm{~d}_{6}\right): \delta(\mathrm{ppm})=$ $165.3,152.2,148.1,141.9,136.3,128.8,126.1,99.5,59.1,53.7,20.6,17.7,14.1$; IR (KBr) $\left(v_{\max } \mathrm{cm}^{-1}\right)$ 3252, 3117, 2980, 1702, 164 7, 1522, 1462, 1228, 1092, 780.

5-Ethoxycarbonyl-4-(3-fluorophenyl)-6-methyl-3,4-dihydropyrimidin-2(1H)-one: $4 i$ M.p. $208-210^{\circ} \mathrm{C},{ }^{1} \mathrm{H}$ NMR $\left(\mathrm{DMSO}_{\mathrm{d}}\right): \delta(\mathrm{ppm})=9.31(\mathrm{~s}, 1 \mathrm{H}, \mathrm{NH}), 7.80(\mathrm{~s}, 1 \mathrm{H}, \mathrm{NH}), 7.34-$ $7.10\left(\mathrm{~m}, 4 \mathrm{H}, \mathrm{C}_{6} H_{4}\right), 5.20(\mathrm{~s}, 1 \mathrm{H}, \mathrm{CH}), 4.10\left(\mathrm{q}, J=6.9 \mathrm{~Hz}, 2 \mathrm{H}, \mathrm{OCH}_{2} \mathrm{CH}_{3}\right), 2.25(\mathrm{~s}, 3 \mathrm{H}$, $\left.\mathrm{CH}_{3}\right), 1.15\left(\mathrm{t}, J=6.9 \mathrm{~Hz}, 3 \mathrm{H}, \mathrm{OCH}_{2} \mathrm{CH}_{3}\right) ;{ }^{13} \mathrm{C} \mathrm{NMR}\left(\mathrm{DMSO}-\mathrm{d}_{6}\right): \delta(\mathrm{ppm})=165.6,164.5$, $160.6,152.4,149.3,148.0,130.9,122.6,114.0,113.5,99.1,59.7,18.2,14.5 ; \mathrm{IR}(\mathrm{KBr})\left(v_{\max } \mathrm{cm}^{-1}\right)$ 3348, 3228, 2935, 2815, 1721, 1637, 1522, 1462,1400, 1288, 1092, 1000, 770.

5-Ethoxycarbonyl-4-(4-hydroxyphenyl)-6-methyl-3,4-dihydropyrimidin-2(1H)-one: $4 j$ M.p. $228-230{ }^{\circ} \mathrm{C},{ }^{1} \mathrm{H}$ NMR $\left(\mathrm{DMSO}_{-} \mathrm{d}_{6}\right): \delta(\mathrm{ppm})=9.18,(\mathrm{~s}, 1 \mathrm{H}, \mathrm{OH}), 8.91(\mathrm{~s}, 1 \mathrm{H}, \mathrm{NH}), 7.79$ $(\mathrm{s}, 1 \mathrm{H}, \mathrm{NH}), 7.19-6.89\left(\mathrm{~m}, 4 \mathrm{H}, \mathrm{C}_{6} H_{4}\right), 5.37(\mathrm{~s}, 1 \mathrm{H}, \mathrm{CH}), 3.99\left(\mathrm{q}, J=7.0 \mathrm{~Hz}, 2 \mathrm{H}, \mathrm{OCH}_{2} \mathrm{CH}_{3}\right)$, $2.41\left(\mathrm{~s}, 3 \mathrm{H}, \mathrm{CH}_{3}\right), 1.05\left(\mathrm{t}, J=7.0 \mathrm{~Hz}, 3 \mathrm{H}, \mathrm{OCH}_{2} \mathrm{CH}_{3}\right) ;{ }^{13} \mathrm{C} \mathrm{NMR}\left(\mathrm{DMSO}-\mathrm{d}_{6}\right): \delta(\mathrm{ppm})=$ 168.5, 158.9, 152.5, 148.3, 136.7, 128.1, 114.3, 110.1, 63.7, 55.5, 15.6, 13.7.

5-Ethoxycarbonyl-4-(2-furyl)-6-methyl-3,4-dihydropyrimidin-2(1H)-one: $4 \mathrm{k}$

M.p. $205-206^{\circ} \mathrm{C},{ }^{1} \mathrm{H}$ NMR $\left(\mathrm{DMSO}_{6}\right): \delta(\mathrm{ppm})=9.26(\mathrm{~s}, 1 \mathrm{H}, \mathrm{N} H), 7.77(\mathrm{~s}, 1 \mathrm{H}, \mathrm{NH}), 7.64(\mathrm{~s}$, $\left.1 \mathrm{H}, \mathrm{CH}_{\text {arom }}\right), 6.36\left(\mathrm{~s}, 1 \mathrm{H}, \mathrm{CH}_{\text {arom }}\right), 6.11\left(\mathrm{~s}, 1 \mathrm{H}, \mathrm{CH}_{\text {arom }}\right), 5.22(\mathrm{~s}, 1 \mathrm{H}, \mathrm{CH}), 4.02(\mathrm{q}, J=7.04 \mathrm{~Hz}$, $2 \mathrm{H}, \mathrm{OCH}_{2} \mathrm{CH}_{3}$ ), 2.43 (s, 3H, $\left.\mathrm{CH}_{3}\right), 1.09$ (t, $\left.J=7.04 \mathrm{~Hz}, 3 \mathrm{H}, \mathrm{OCH}_{2} \mathrm{CH}_{3}\right) ;{ }^{13} \mathrm{C}$ NMR (DMSO$\left.\mathrm{d}_{6}\right): \delta(\mathrm{ppm})=165.9,156.2,152.8,150.0,142.6,110.7,105.7,97.0,58.0,51.3,20.9,18.1$; IR $(\mathrm{KBr})\left(v_{\max } \mathrm{cm}^{-1}\right) 3317,3116,2925,1725,1639,1431,1342,1238,1087,761$. 


\section{5-Acetyl-4-(2-methoxyphenyl)-6-methyl-3,4-dihydropyrimidin-2(1H)-one: $4 \mathrm{l}$}

M.p. $252-254^{\circ} \mathrm{C},{ }^{1} \mathrm{H}$ NMR $\left(\mathrm{DMSO}_{\mathrm{d}}\right): \delta(\mathrm{ppm})=9.17(\mathrm{~s}, 1 \mathrm{H}, \mathrm{NH}), 7.38(\mathrm{~s}, 1 \mathrm{H}, \mathrm{NH}), 6.88-$ $7.25\left(\mathrm{~m}, 4 \mathrm{H}, \mathrm{C}_{6} H_{4}\right), 5.50(\mathrm{~s}, 1 \mathrm{H}, \mathrm{CH}), 3.80\left(\mathrm{~s}, 3 \mathrm{H}, \mathrm{OCH}_{3}\right), 2.26\left(\mathrm{~s}, 3 \mathrm{H}, \mathrm{COCH}_{3}\right), 2.02(\mathrm{~s}, 3 \mathrm{H}$, $\left.\mathrm{CH}_{3}\right) .{ }^{13} \mathrm{C}$ NMR $\left(\mathrm{DMSO}-\mathrm{d}_{6}\right): \delta(\mathrm{ppm})=195.0,156.7,152.6,148.6,131.5,129.4,127.2$, 120.8, 111.7, 108.2, 55.8, 49.1, 30.1, 19.1; IR (KBr) $\left(v_{\max } \mathrm{cm}^{-1}\right) 3224,3109,2929,2848$, $1679,1602,1461,1436,1384,1321,1278,1092,759$.

\section{5-Acetyl-6-methyl-4-(3-methyphenyl)-3,4-dihydropyrimidin-2(1H)-one: $4 \mathrm{~m}$}

M.p. $256-258^{\circ} \mathrm{C},{ }^{1} \mathrm{H}$ NMR $\left(\mathrm{DMSO}_{6}\right): \delta(\mathrm{ppm})=9.18(\mathrm{~s}, 1 \mathrm{H}, \mathrm{N} H), 7.82(\mathrm{~s}, 1 \mathrm{H}, \mathrm{NH}), 7.25-$ $7.10\left(\mathrm{~m}, 4 \mathrm{H}, \mathrm{C}_{6} H_{4}\right), 5.25(\mathrm{~s}, 1 \mathrm{H}, \mathrm{CH}), 2.30\left(\mathrm{~s}, 6 \mathrm{H}, \mathrm{C}_{6} \mathrm{H}_{4}-\mathrm{CH}_{3}, \mathrm{COCH}_{3}\right), 2.15\left(\mathrm{~s}, 3 \mathrm{H}, \mathrm{CH}_{3}\right)$; ${ }^{13} \mathrm{C}$ NMR (DMSO-d $\left.\mathrm{d}_{6}\right): \delta(\mathrm{ppm})=195.0,152.5,148.0,145.0,137.5,130.0,129.5,127.5$, 125.0, 110.0, 54.0, 30.0, 22.0, 18.5; IR (KBr) $\left(v_{\max } \mathrm{cm}^{-1}\right) 3368,3122,2931,2734,1706$, $1595,1382,1330,786$.

5-Acetyl-6-methyl-4-(2-nitrophenyl)-3,4-dihydropyrimidin-2(1H)-one: $4 n$

M.p. $235-237^{\circ} \mathrm{C},{ }^{1} \mathrm{H}$ NMR $\left(\mathrm{DMSO}_{\mathrm{d}}\right): \delta(\mathrm{ppm})=9.38(\mathrm{~s}, 1 \mathrm{H}, \mathrm{NH}), 8.13-8.11(\mathrm{~m}, 2 \mathrm{H}$, $\left.\mathrm{CH}_{\text {arom }}\right), 7.91(\mathrm{~s}, 1 \mathrm{H}, \mathrm{NH}), 7.66-7.61\left(\mathrm{~m}, 2 \mathrm{H}, \mathrm{CH}_{\text {arom }}\right), 5.29(\mathrm{~s}, 1 \mathrm{H}, \mathrm{CH}), 2.26(\mathrm{~s}, 3 \mathrm{H}$, $\left.\mathrm{COCH}_{3}\right), 2.15\left(\mathrm{~s}, 3 \mathrm{H}, \mathrm{CH}_{3}\right) ;{ }^{13} \mathrm{C}$ NMR $\left(\mathrm{DMSO}_{-} \mathrm{d}_{6}\right): \delta(\mathrm{ppm})=196.5,156.8,147.0,137.5$, 137.0, 134.4, 128.0, 127.4, 123.4, 115.4, 39.1, 22.6, 17.3.

\section{5-Acetyl-4-(4-methoxylphenyl)-6-methyl-3,4-dihydropyrimidin-2(1H)-one: 4o}

M.p. $182-183{ }^{\circ} \mathrm{C},{ }^{1} \mathrm{H}$ NMR $\left(\mathrm{DMSO}_{6}\right): \delta(\mathrm{ppm})=9.17(\mathrm{~s}, 1 \mathrm{H}, \mathrm{NH}), 7.78(\mathrm{~s}, 1 \mathrm{H}, \mathrm{NH}), 7.19-$ $7.13\left(\mathrm{~m}, 2 \mathrm{H}, \mathrm{CH} \mathrm{H}_{\text {arom }}\right), 6.91-6.85\left(\mathrm{~m}, 2 \mathrm{H}, \mathrm{CH}_{\text {arom }}\right), 5.21(\mathrm{~s}, 1 \mathrm{H}, \mathrm{CH}), 3.74\left(\mathrm{~s}, 3 \mathrm{H}, \mathrm{COCH}_{3}\right)$, $2.29\left(\mathrm{~s}, 3 \mathrm{H}, \mathrm{OCH}_{3}\right), 2.09\left(\mathrm{~s}, 3 \mathrm{H}, \mathrm{CH}_{3}\right) ;{ }^{13} \mathrm{C} \mathrm{NMR}\left(\mathrm{DMSO}-\mathrm{d}_{6}\right): \delta(\mathrm{ppm})=195.0,158.9$, 152.6, 148.4, 136.7, 128.1, 114.3, 110.1, 55.5, 53.7, 30.6, 19.3.

\section{5-Acetyl-6-methyl-4-(2-thienyl)-3,4-dihydropyrimidin-2(1H)-one: $4 p$}

M.p. $223-224^{\circ} \mathrm{C},{ }^{1} \mathrm{H}$ NMR $\left(\mathrm{DMSO}_{\mathrm{d}}\right): \delta(\mathrm{ppm})=9.36(\mathrm{~s}, 1 \mathrm{H}, \mathrm{NH}), 8.01(\mathrm{~d}, 1 \mathrm{H}, \mathrm{NH}), 7.37-$ $7.35\left(\mathrm{~m}, 1 \mathrm{H}, \mathrm{C} H_{\text {arom }}\right), 7.01-6.93\left(\mathrm{~m}, 2 \mathrm{H}, \mathrm{CH} H_{\text {arom }}\right), 5.54(\mathrm{~d}, 1 \mathrm{H}, \mathrm{CH}), 2.35\left(\mathrm{~s}, 3 \mathrm{H}, \mathrm{COCH}_{3}\right)$, $2.13\left(\mathrm{~s}, 3 \mathrm{H}, \mathrm{CH}_{3}\right) ;{ }^{13} \mathrm{C}$ NMR $\left(\mathrm{DMSO}_{-} \mathrm{d}_{6}\right): \delta(\mathrm{ppm})=194.3,152.7,149.0,148.7,127.2$, 125.3, 124.4, 110.9, 49.6, 30.6, 19.3.

\section{Results and Discussion}

We would like to disclose here our preliminary results using the inexpensive, easily available and recovered catalyst, $\mathrm{Pb}\left(\mathrm{NO}_{3}\right)_{2}$ for the preparation of 3,4-dihydropyrimidin-2(1H)-ones.

It is noted that this catalyst is the first derivative of lead employed to promote the Biginelli reaction. Therefore, it will be the leader file of other lead derivatives which have never been the subject of investigation up to now.

In order to improve yields, some experimentation with respect to the molar ratio of reactants and the nature of the solvent were examined. The best results to produce good to excellent yields (70-96\%) of dihydropyrimidinone $\mathbf{4}$, were achieved on using a 1/1.5/1 molar ratio of aldehyde 1, urea 2 and 1,3-dicarbonyl compound 3 in the presence of $5 \mathrm{~mol} \%$ of $\mathrm{Pb}\left(\mathrm{NO}_{3}\right)_{2}$ in one-pot condensation employing refluxing $\mathrm{CH}_{3} \mathrm{CN}$ (Scheme 1). The results are summarized in Table 1. Apparently, under these conditions, the nature of the substituent in the aromatic moiety does not affect significantly the yield of the reactions. As can be seen from data in Table 1, in all cases studied, the three-component reaction with both aromatic aldehydes carrying either electron-donating or electron-withdrawing substituents, heteroaromatic aldehydes and even alkylsubstituted aromatic aldehydes, proceeded smoothly giving the corresponding dihydropyrimidinones in high yields. 
Further, instead of ethyl acetoacetate, acetyl acetone was used as the 1,3-dicarbonyl compound without loss of efficiency (Table 1, products: 4l-p).

Table 1. $\mathrm{Pb}\left(\mathrm{NO}_{3}\right)_{2}$ catalysed synthesis of Biginelli 3,4-dihydropyrimidinones.

\begin{tabular}{|c|c|c|c|c|c|c|c|}
\hline \multirow{2}{*}{ DHPM $4^{\mathrm{a}}$} & \multirow{2}{*}{$\mathrm{R}$} & \multirow{2}{*}{$\mathrm{R}_{1}$} & \multirow{2}{*}{ Time, min } & \multicolumn{2}{|c|}{ Yield, \% } & \multicolumn{2}{|c|}{ M.P, ${ }^{\circ} \mathrm{C}$} \\
\hline & & & & $A^{b}$ & $\mathrm{~B}^{\mathrm{c}}$ & Found $^{\mathrm{d}}$ & Reported \\
\hline $4 a$ & $\mathrm{C}_{6} \mathrm{H}_{5}$ & OEt & 30 & 96 & $78^{49}$ & $206-207$ & $206-207^{17}$ \\
\hline $4 b$ & $2-\mathrm{Me}-\mathrm{C}_{6} \mathrm{H}_{4}$ & OEt & 95 & 89 & & $202-204$ & $208-210^{38}$ \\
\hline $4 \mathrm{c}$ & $2-\mathrm{MeO}-\mathrm{C}_{6} \mathrm{H}_{4}$ & OEt & 30 & 84 & & $258-259$ & $257-259^{37}$ \\
\hline $4 d$ & $(3-\mathrm{HO}, 4-\mathrm{MeO})-\mathrm{C}_{6} \mathrm{H}_{3}$ & OEt & 60 & 60 & & $186-188$ & $185-187^{36}$ \\
\hline $4^{\mathrm{e}}$ & $3-\mathrm{Me}-\mathrm{C}_{6} \mathrm{H}_{4}$ & OEt & 10 & 75 & & $228-230$ & \\
\hline $4 f$ & $4-\mathrm{MeO}-\mathrm{C}_{6} \mathrm{H}_{4}$ & OEt & 180 & 89 & $61^{49}$ & $200-202$ & $200-201^{36}$ \\
\hline $4 \mathrm{~g}$ & $3-\mathrm{Cl}-\mathrm{C}_{6} \mathrm{H}_{4}$ & OEt & 08 & 70 & $56^{50}$ & $190-193$ & $193-195^{38}$ \\
\hline $4 \mathrm{~h}$ & $4-\mathrm{Me}-\mathrm{C}_{6} \mathrm{H}_{4}$ & OEt & 10 & 69 & & $213-215$ & $215-216^{38}$ \\
\hline $4 \mathrm{i}$ & $3-\mathrm{F}-\mathrm{C}_{6} \mathrm{H}_{4}$ & OEt & 140 & 96 & & $208-210$ & \\
\hline $4 j$ & 4-HO- $\mathrm{C}_{6} \mathrm{H}_{4}$ & OEt & 40 & 70 & $67^{49}$ & $230-232$ & $228-230^{39}$ \\
\hline $4 \mathrm{k}$ & 2-Furyl & OEt & 90 & 70 & $36^{49}$ & $205-207$ & $203-205^{36}$ \\
\hline $4 l$ & $2-\mathrm{MeO}-\mathrm{C}_{6} \mathrm{H}_{4}$ & $\mathrm{Me}$ & 60 & 72 & & $252-254$ & \\
\hline $4 \mathrm{~m}$ & $3-\mathrm{Me}-\mathrm{C}_{6} \mathrm{H}_{4}$ & $\mathrm{Me}$ & 22 & 70 & & $256-258$ & - \\
\hline $4 n$ & $2-\mathrm{NO}_{2}-\mathrm{C}_{6} \mathrm{H}_{4}$ & $\mathrm{Me}$ & 15 & 70 & & $234-236$ & - \\
\hline 40 & $4-\mathrm{MeO}-\mathrm{C}_{6} \mathrm{H}_{4}$ & $\mathrm{Me}$ & 90 & 88 & & $182-184$ & $178-180^{15}$ \\
\hline $4 p$ & 2-Thienyl & $\mathrm{Me}$ & 120 & 72 & & $223-224$ & \\
\hline
\end{tabular}

${ }^{a}$ All products were characterized by ${ }^{1} \mathrm{H},{ }^{13} \mathrm{C} N \mathrm{~N}$ and $\mathrm{IR}$ spectroscopy.

${ }^{b}$ Method A: using our new conditions (cat. $\mathrm{Pb}\left(\mathrm{NO}_{3}\right)_{2}$ in $\mathrm{CH}_{3} \mathrm{CN}$, Reflux).

${ }^{c}$ Method B: Classical Biginelli conditions (cat. HCl in EtOH, reflux 18h)

${ }^{d}$ Melting points are uncorrected.

For comparison purposes, yields obtained for $\mathbf{4 a}, \mathbf{4 f}, \mathbf{4 g}, \mathbf{4 j}$ and $\mathbf{4 k}$ using the traditional Biginelli conditions $(\mathrm{EtOH} / \mathrm{HCl}$, reflux, method $\mathrm{B})$ are given in Table 1. As can be seen, the present method $\left(\mathrm{PbN}\left(\mathrm{O}_{3}\right)_{2} / \mathrm{CH}_{3} \mathrm{CN}\right.$, reflux, method $\left.\mathrm{A}\right)$ produced higher yields in shorter reaction times than the classical Biginelli method.

\section{Conclusions}

In conclusion, we have developed an efficient and simple method for the direct preparation of substituted 3,4-dihydropyrimidin-2-( $1 \mathrm{H})$-ones via the Biginelli reaction using $\mathrm{Pb}\left(\mathrm{NO}_{3}\right)_{2}$ as a recovered catalyst in good yields and short reaction times from readily available starting materials.

\section{Acknowledgements}

We gratefully acknowledge the MESRES (Ministère de l'Enseignement Supérieur et de la Recherche Scientifique) for financial support.

\section{References}

1. Biginelli P, Gazz Chim Ital. 1893, 23, 360 .

2. (a) Kappe C O, Tetrahedron, 1993, 49, 6837; (b) Kappe C O, Acc Chem Res., 2000, 33, 879.

3. Myer T U, Kappoor T M, Haggarty S J, King R W, Schreiber S I and Mitchison T J, Science, 1999, 286, 971.

4. Sadanadam Y S, Shetty M M and Diwan P V, Eur. J Med Chem., 1992, 27, 87. 
5. Hurst E W and Hull, R, J Med Pharm Chem., 1961, 3, 215.

6. Kappe C O, Shishkin O V, Uray G and Verdino P, Tetrahedron, 2000, 56, 1859.

7. (a) Atwal K S, Rovnyak G C, O'Reilly B C and Schwartz J J, J. Org. Chem., 1989, 54, 5898; (b) Kappe C O, Fabian W M F and Semones M A, Tetrahedron, 1997, 53, 2803.

8. Atwal K S, Rovnyak G C , Schwartz J, Moreland S, Hedberg A, Gougoutas J Z, Malley M F and Floyd D M, J Med Chem., 1990, 33, 1510.

9. Horovitz Z P (E.R. Squibb, and Sons), Eur Pat Appl., 1990, EP 400, 665 [Chem. Abstr., 1991, 115, 64793].

10. Crosson C E, Potter D E, Ondetti M A, Floyd D and Aberg G, (Houston Biotechnology, Inc.; Squibb E R, and Sons) PCT Int. Appl. WO 1990, 06,118 [Chem. Abstr., 1991, 114,157224 w].

11. Stefani H A, Oliveira C B, Almeida R B, Pereira C M P, Braga R C, Cella R, Borges V C, Savegnago L and Nogueira C W, Eur J Chem., 2006, 41, 513.

12. Patil A D, Kumar N V, Kokke W C, Bean M F, Freyer A J, De brosse C, Mai S, Truneh A, Faulkner D J, Carte B, Breen A L, Hertzberg R P, Johnson R K, Westly J W and Potts B C, J Org Chem., 1995, 60, 1182.

13. Overman L E, Robinowitz M H and Renhowe P A, J Am Chem Soc., 1995, 117, 2657.

14. (a) Verale R, Alam M M and Adapa S R, Synlett., 2003, 67; (b) Lu J, Bai Y, Wang Z J, Yang B and Ma H, Tetrahedron Lett., 2000, 41, 9075; (c) Reddy C V, Mahesh M, Raju PV, Babu T and Reddy V V, Tetrahedron Lett., 2002, 43, 3657; (e) Xia M, Wang Y, Tetrahedron Lett., 2002, 43, 7703; (f) Jin S T, Wang H X, Xing C Y, Li X L and Li T S, Synth Commun., 2004, 34, 3009; (g) Ghosh R, Maiti S and Chacraborty A, J Mol Catal A: Chem., 2004, 217, 47; (h) Zhu Y, Pan Y and Huang S, Synth Commun., 2004, 34, 3167 and references cited therein.

15. Ma Y, Qian C T, Wang L M and Yang M, J Org Chem., 2000, 65, 3864.

16. Brindban A and Jana J U, Org Chem., 2000, 65, 6270.

17. Salitha G, Reddy G S K and Yadav J S, Tetrahedron Lett., 2003, 44, 6497.

18. Gohain M, Prajapati D and Sandhu J S, Synlett., 2004, 235.

19. Maiti G, Kundu P and Guin C, Tetrahedron Lett., 2003, 44, 2757.

20. Yadav J S, Reddy B V S, Srinivas R, Venugopal C and Ramalingam T, Synthesis, 2001, 1341.

21. SuryaK De and Gibbs A R, Synthesis, 2005, 11, 1748.

22. Russowsky D, Lopes F A, da Silva V S S, Canto K F S, Montes D’Oca M G and Godoi M N, J Braz Chem Soc., 2004, 165.

23. Hu E H, Silder D R and Dolling U H, J Org Chem., 1998, 63, 3454.

24. Rodríguez- Domínguez J C, Bernardi D and Kirsch G, Tetrahedron Lett., 2007, 48, 5777.

25. Nandurkar N S, Bhanushalia M J, Bhora M D and Bhanage B M, J Mol Catal A: Chem, 2007, 271, 14.

26. Paraskar A S, Dewkar G K and Sudalai A, Tetraheron Lett., 2003, 44, 3305.

27. Kalita H R and Phukan P, Catal Commun., 2007, 8, 179.

28. Fu N, Yuan Y, Cao Z, Wang S, Wang J and Peppe C, Tetrahedron, 2002, 58, 4801.

29. Tu S J, Fang F, Miao C B, Jiang H, Shi D Q and Wang X S, Tetrahedron Lett., 2003, 44, 6153.

30. Ghosh R, Maiti S and Chakraborty A, J Mol Cat., 2004, 47, 217.

31. Debache A, Boumoud B, Amimour M, Belfaitah A, Rhouati S and Carboni B, Tetrahedron Lett., 2006, 47, 5697. 
32. Adibi H, Samimi K A and Beygzadeh M, Catal Commun., 2007, 8, 2119.

33. Chen W Y, Qin S D and Jin J R, Catal Commun., 2007, 8, 123.

34. Jin T, Zhang S and Li T, Synth Commun., 2002, 32, 1847.

35. Salehi P, Dabiri M, Zolfigol M A and Fard M A B, Tetrahedron Lett., 2003, 44, 2889.

36. TU S, Fang F, Zhu S L, Zhang T X and Zhuang Q, Synlett., 2004, 53.

37. Jiang C and Qi Dong You, Chin Chem Lett. 2007, 18, 647.38.

38. Yu Y, Liu D, Liu C and Lu G, Bioorg Med Chem Lett., 2007, 17, 3508.

39. Heravi M M, Bakhtiari K and Bamoharram F Z, Catal Commun., 2006, 7, 373.

40. Maradur S P and Gokavi G S, Catal Commun., 2007, 8, 279.

41. Kumar A and Maurya R A, Tetrahedron Lett., 2007, 48, 4569.

42. (a) Munoz-Muniz O and Juaristi E, Arkivoc, 2003, 9, 16; (b) Huang Y, Yang F and Zhu C, J Am Chem Soc., 2005, 127, 16386.

43. Peng J and Den Y g, Tetrahedron Lett., 2001, 42, 5917.

44. Wipf P and Cunningham A, Tetrahedron Lett., 1995, 36, 7819.

45. Studer A, Jeger P, Wipf P and Curran D P, J Org Chem., 1997, 62, 2817.

46. Li J T, Han J F, Yang J H and Li T S, Ultrasonics Sonochemistry, 2003, 10, 119.

47. Ranu B C, Hajra A and Jana U, J Org Chem., 2000, 65, 6270.

48. Lu J, Bai Y, Yang B and Ma H, Tetrahedron Lett., 2000, 41, 9075.

49. Folkers K, Harwood H J and Johnson T B, J Am Chem Soc., 1932, 54, 3751.

50. Lu J and Ma H, Synlett., 2000, 63. 


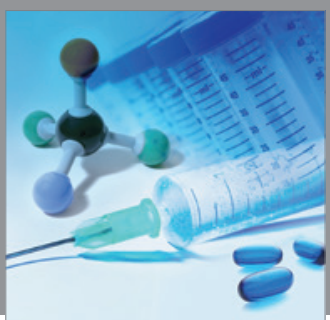

International Journal of

Medicinal Chemistry

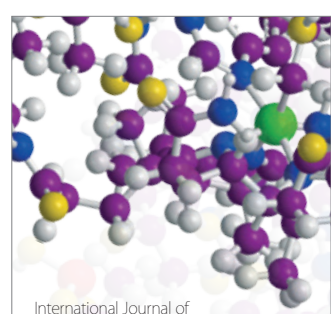

Carbohydrate Chemistry

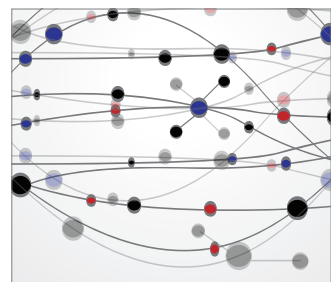

The Scientific World Journal
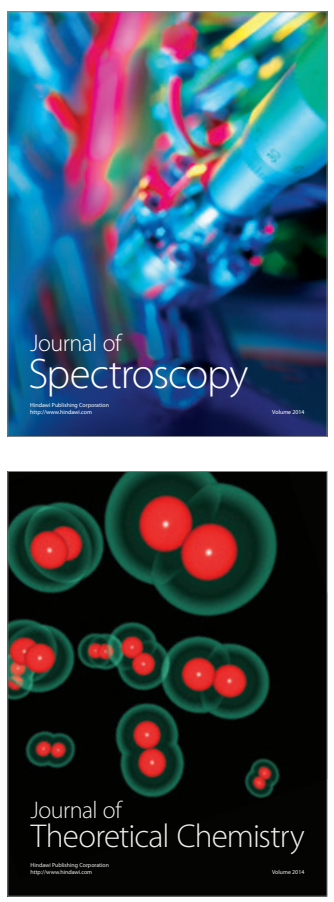
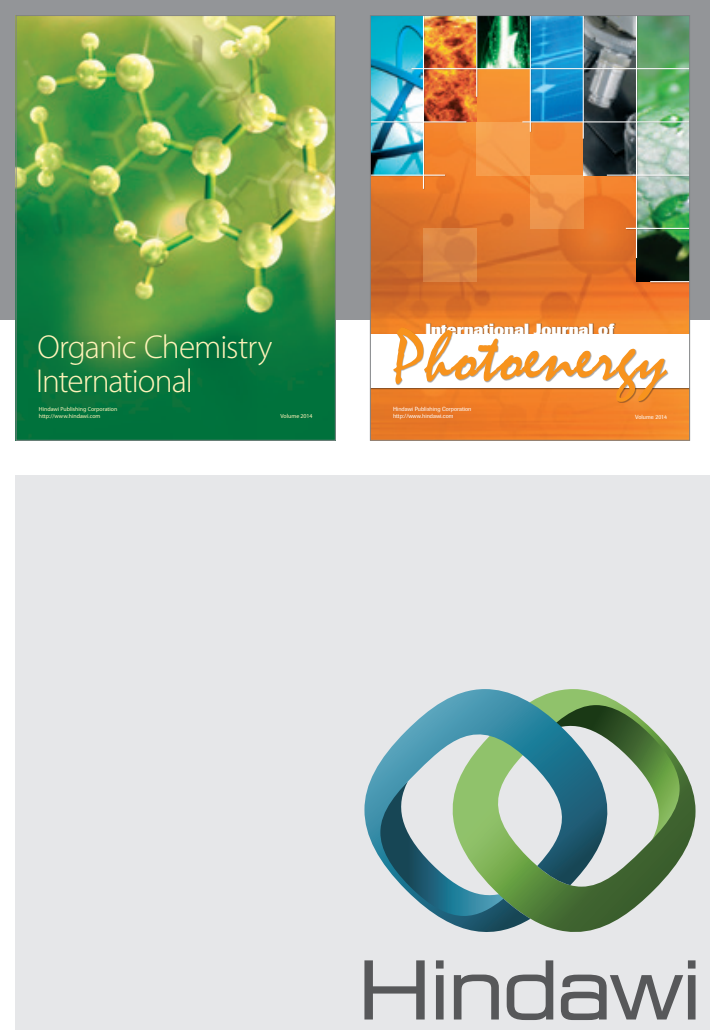

Submit your manuscripts at

http://www.hindawi.com
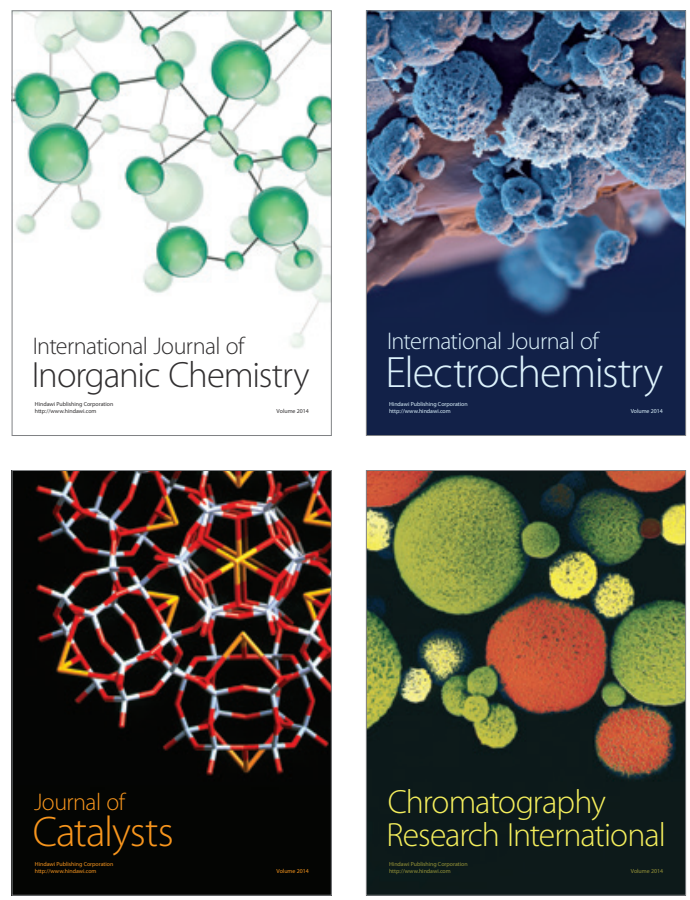
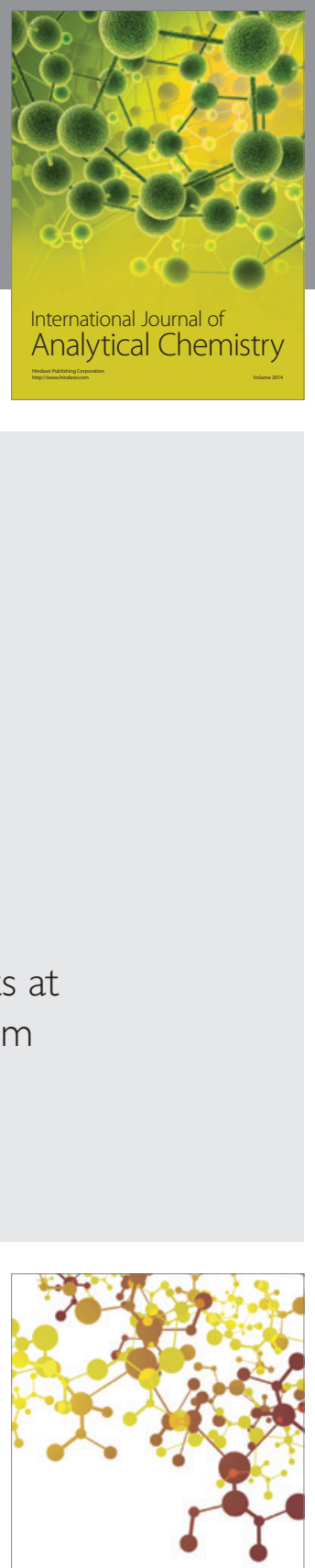

Journal of

Applied Chemistry
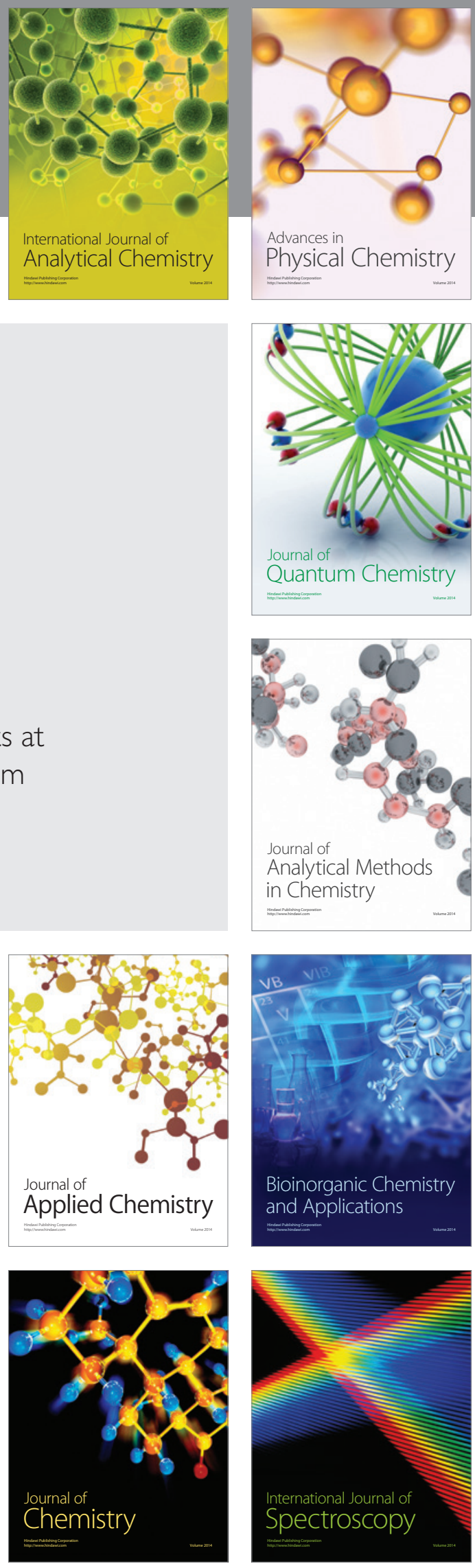